\title{
聚苯胺炭柱撑石墨烯复合材料的制备及其电化学性能的研究
}

\author{
胡 茜 $^{1}$, 刘洪波 ${ }^{1,2}$, 夏笑虹 ${ }^{1,2}$, 谷智强 ${ }^{1}$
}

(湖南大学 1. 材料科学与工程学院; 2. 先进炭材料及应用技术湖南省重点实验室, 长沙 410082)

摘 要: 通过真空抽滤诱导自组装及热解还原处理, 制备出具有柱撑结构的聚苯胺炭/石墨烯复合材料(PGR)。采 用 $X$ 射线衍射 $(X R D)$ 、透射电子显微镜(TEM)、X 射线电子能谱(XPS)和电化学测试等表征技术考察了聚苯胺单 体(AN)与氧化石墨烯(GO)质量比对 PGR 结构和电化学性能的影响。结果表明, 聚苯胺炭均匀分布在石墨烯(GR) 片层间形成三维导电网络, 有效地增大了 GR 的层间距, 且实现了氮掺杂, 显著提高了 GR 的结构稳定性和电化 学性能; $\mathrm{AN}$ 与 $\mathrm{GO}$ 质量比为 $1: 1$ 时制备的样品 PGR 1 在 $100 \mathrm{~mA} / \mathrm{g}$ 电流密度下的首次脱锂比容量为 $653 \mathrm{mAh} / \mathrm{g}$, 当 电流密度增大至 $1 \mathrm{~A} / \mathrm{g}$ 时, 仍具有高达 $343 \mathrm{mAh} / \mathrm{g}$ 的脱锂比容量, 远高于 $\mathrm{GR}$ 的脱锂比容量 $(101 \mathrm{mAh} / \mathrm{g})$, 表现出 优异的倍率性能。

关 键 词: 石墨烯; 聚苯胺; 柱撑结构; 锂离子电池; 负极材料

中图分类号: TM911 文献标识码: A

\section{Polyaniline-carbon Pillared Graphene Composite: Preparation and Electrochemical Performance}

\author{
HU Xi ${ }^{1}$, LIU Hong-Bo ${ }^{1,2}$, XIA Xiao-Hong ${ }^{1,2}$, GU Zhi-Qiang ${ }^{1}$
}

(1. College of Material Science and Engineering, Hunan University, Changsha 410082, China; 2. Hunan Province Key Laboratory for Advanced Carbon Materials and Applied Technology, Hunan University, Changsha 410082, China)

\begin{abstract}
Polyaniline-carbon pillared graphene composites (PGR) were successfully prepared by vacuum extraction induced self-assembly and pyrolysis method. Effects of the mass ratio of aniline monomer (AN) and graphene oxide (GO) on structure and electrochemical properties of PGR were investigated by X-ray diffraction, transmission electron microscopy, X-ray photoelectron spectroscopy, and electrochemical characterization. Results showed that the polyaniline-carbon pillars uniformly distributed between the graphene (GR) layers to form a three-dimensional conductive network with expanded interlayer space and nitrogen doping, which effectively improved the structural stability and electrochemical performance of GR. The as-prepared PGR with the mass ratio of AN and GO at $1: 1$ exhibits a high reversible capacity of $653 \mathrm{mAh} / \mathrm{g}$ at a current density of $100 \mathrm{~mA} / \mathrm{g}$ and an excellent rate capability of $343 \mathrm{mAh} / \mathrm{g}$ at a current density of $1 \mathrm{~A} / \mathrm{g}$, all that is much higher than that of the GR electrode $(101 \mathrm{mAh} / \mathrm{g})$.
\end{abstract}

Key words: graphene; polyaniline; pillared structure; lithium-ion batteries; anode material

石墨烯具有导电率高 $\left(10^{6} \mathrm{~S} / \mathrm{m}\right)$ 、电子迁移率大 $\left(10^{4} \mathrm{~cm}^{2} /(\mathrm{V} \cdot \mathrm{s})\right)$ 等特点 ${ }^{[1-2]}$, 自 2004 年被发现以来人
们一直希望能将其用作锂离子电池负极材料。但石 墨烯的比表面积非常高 $\left(2630 \mathrm{~m}^{2} / \mathrm{g}\right)$ ，直接用作负极

收稿日期：2018-05-02; 收到修改稿日期：2018-05-30

基金项目: 国家自然科学基金(51772083, 51402101); 湖南省自然科学基金(14JJ3059, 2017JJ2008)

National Natural Science Foundation of China (51772083, 51402101); Hunan Provincial Natural Science Foundation of China (14JJ3059, 2017JJ2008)

作者简介: 胡 茜(1993-), 女, 硕士研究生. E-mail: xihuzwc@163.com

通讯作者: 刘洪波，教授. E-mail: hndxlhb@163.com 
材料存在不可逆容量大，首次库伦效率低等问题。 此外, 石墨烯较大的共轭平面导致其容易发生无序 堆叠, 使其在充放电循环过程中结构不稳定, 优异 性能无法充分发挥 ${ }^{[3-6]}$ 。为此, 研究者提出了很多改 性方法来阻止石墨烯的团聚, 主要包括膨胀法 ${ }^{[6-7]}$ 、 有序组装 ${ }^{[8-9]}$ 和加入隔离物 ${ }^{[10-11]}$ 等。

氧化石墨经过热膨胀后，原本聚集的氧化石墨 烯片层被剥离和还原，这种方法可以有效制备多孔 石墨烯材料, 但结构可控性较差 ${ }^{[12-14]}$ 。有序组装法 是将石墨烯片通过可控的组装技术，制备出有序堆 叠且具有多孔网络结构的石墨烯材料 ${ }^{[15-18]}$, 但结构 稳定性较差。在石墨烯片层中加入纳米材料将其隔 开的方法, 则可以利用纳米材料与石墨烯片层的化 学结合提高石墨烯有序堆叠结构的稳定性, 进而提 高其电化学性能。现已报道的纳米材料包括金属氧 化物、碳纳米管等 ${ }^{[19-20]}$ 。

聚苯胺(PANI)经质子酸掺杂后呈正电性, 且分 子链上存在共轭基团, 容易与氧化石墨烯或石墨烯 通过静电吸引、 $\pi-\pi$ 共轭以及氢键作用而有序组装 成聚苯胺柱撑氧化石墨烯结构 ${ }^{[21-22]}$ 。经高温热解后 形成的聚苯胺炭/石墨烯复合材料, 既可利用聚苯胺 炭的柱撑作用, 在石墨烯片层中增加储锂空间、改 善结构稳定性, 又可实现石墨烯的氮掺杂, 在石墨 烯中形成更多的活性位点, 改善其电子性能 ${ }^{[23]}$ 。因 而有望提高石墨烯的电化学性能。

本研究结合热膨胀法、有序组装、添加隔离物 三种石墨烯改性方式的优点, 利用氧化石墨烯(GO) 丰富的化学修饰和诱导自组装特性, 以及易于与其 它材料通过共价、非共价作用进行复合的特点, 以 苯胺(AN)为单体, 通过原位聚合、真空抽滤诱导自 组装、热解还原等工艺制备出具有柱撑结构的聚苯 胺炭/石墨烯复合材料。考察了 $\mathrm{AN}$ 与 GO 质量比对 聚苯胺炭柱撑石墨烯复合材料结构和电化学性能的 影响，通过对比分析，阐明了倍率性能提高的原因。

\section{1 实验方法}

\section{1 聚苯胺炭柱撑石墨烯复合材料的制备}

采用改进 Hummers 法 ${ }^{[24]}$ 制备氧化石墨烯, 超声 并离心处理得到 $\mathrm{GO}$ 。取适量 $\mathrm{GO}$ 溶液 $(1 \mathrm{mg} / \mathrm{mL})$, 加 入一定量 $\mathrm{AN}$, 利用氨水调节 $\mathrm{pH}$ 至 10 , 超声 $1 \mathrm{~h}$ 后, 得到含 $\mathrm{GO}$ 和 $\mathrm{AN}$ 的混合溶液。将适量过硫酸铵 (与 $\mathrm{AN}$ 的摩尔比为 $1: 1$ ) 溶解在 $1 \mathrm{~mol} / \mathrm{L}$ 的 $\mathrm{HCl}$ 溶液中, 再加入混合溶液，在 $0 \sim 5{ }^{\circ} \mathrm{C}$ 下持续搅拌反应 $6 \mathrm{~h}$ 。静 置一定时间后进行抽滤, 并用去离子水和酒精依次 洗涤至滤液呈中性, 冷冻干燥后得到聚苯胺/氧化石
墨烯复合材料(PGO)。将 PGO 置于行星球磨机的氧 化锆球磨罐中, 按照球料比为 $10: 1$ 加入氧化锆球 后, 以 $450 \mathrm{r} / \mathrm{min}$ 的转速球磨 $6 \mathrm{~h}$ 得到粉状 $\mathrm{PGO}$ 。将 粉状 PGO 于 $195^{\circ} \mathrm{C}$ 空气中预氧化 $2 \mathrm{~h}$ 后, 再在 $\mathrm{N}_{2}$ 保护下以 $2{ }^{\circ} \mathrm{C} / \mathrm{min}$ 的速率升温至 $800{ }^{\circ} \mathrm{C}$, 并保温 $2 \mathrm{~h}$, 自然冷却至室温后即得到具有柱撑结构的 PGR 复 合材料。制备过程如图 1 所示。作为对比，按上述 步骤制备石墨烯 $(\mathrm{GR})$ 、不含石墨烯的纯 PANI 和纯 聚苯胺炭 $(\mathrm{CP})$ 。为方便计, 将 $\mathrm{AN}$ 与 $\mathrm{GO}$ 质量比分别 为 $0.5 、 1$ 的聚苯胺/氧化石墨烯复合材料和聚苯胺炭 /石墨烯复合材料分别简记为 PGO0.5、PGO1 和 PGR0.5、PGR1。并将 $\mathrm{AN}$ 与 $\mathrm{GO}$ (质量比为 $1: 1$ ) 通 过直接吸附 (未添加引发剂和未经炭化过程)形成的 产物简记为 $\mathrm{AGO} 1$ 。

\section{2 分析表征}

利用扫描电子显微镜(SEM, S-4800, Hitachi)与 透射电子显微镜(TEM, JEM3010)观察样品形貌, 采 用 $\mathrm{X}$ 射线衍射仪(XRD, D8-advance)、红外光谱分析 仪(FT-IR, Nicolet Nexus 470)、拉曼光谱仪(Raman, Labram-010)和 X 射线光电子能谱(XPS, Kratos Analytical)对材料的结构与组分进行分析, 并通过比 表面积测试仪(Tri-star 3020 analyzer)测试样品的比 表面积与孔径分布。

\section{3 电化学性能测试}

按质量比 $8: 1: 1$ 将活性物质、聚偏氟乙烯 (PVDF)和乙炔黑混合均匀, 以 $N$-甲基吡咯烷酮为 溶剂配成浆料。将浆料涂覆在铜箔上，放入真空干 燥箱中 $80^{\circ} \mathrm{C}$ 干燥 $12 \mathrm{~h}$ 后, 制成直径为 $14 \mathrm{~mm}$ 的电 极片 (其中活性物质的质量约为 $1 \mathrm{mg}$ )。以金属锂片 为对电极, 电解液为 $1 \mathrm{~mol} / \mathrm{L} \mathrm{LiPF}_{6}$ 的碳酸乙酯(EC)/

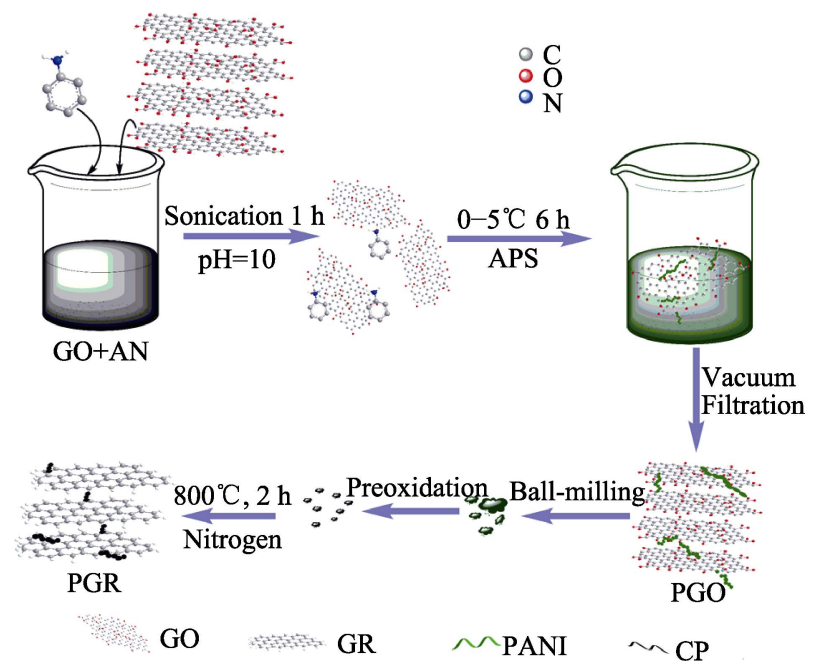

图 1 PGR 复合材料的制备过程示意图

Fig. 1 Schematic illustration of the preparation of PGR composites 
碳酸二甲酯(DMC)/碳酸二乙酯(DEC)的混合液（体 积比为 1/1/1), 隔膜为 Celgard 2300 聚丙烯膜, 在 手套箱中组装成 CR2016 型扣式电池。

采用 CT2100A 型 LAND 电池测试系统对电池进 行恒电流充放电测试, 电流密度为 $100 \mathrm{~mA} / \mathrm{g} \sim 1 \mathrm{~A} / \mathrm{g}$, 测试电压为 $2.0 \sim 0.001 \mathrm{~V}\left(v s . \mathrm{Li}^{+} / \mathrm{Li}\right)$ 。利用 $\mathrm{CHI} 660 \mathrm{E}$ 型电化学工作站进行循环伏安(CV)和交流阻抗(EIS) 测试, CV 测试电压为 $2.0 \sim 0.001 \mathrm{~V}\left(v s . \mathrm{Li}^{+} / \mathrm{Li}\right)$, 扫描 速率为 $0.1 \mathrm{mV} / \mathrm{s}$; EIS 测试电压幅度为 $10 \mathrm{mV}$, 频率 为 $0.01 \sim 100 \mathrm{kHz}$ 。

\section{2 结果与讨论}

\section{1 结构与形貌}

图 2 是原料及不同制备过程中所得产物的 XRD 图谱，包括 GO、AGO1、PGO0.5、PGO1、GR、PGR0.5 与 $\mathrm{PGR} 1$ 。从图 2 可以看出, $\mathrm{GO}$ 在 $2 \theta=12.50^{\circ}$ 存在明 显的衍射峰，归属于 $\mathrm{GO}$ 的(001)面，对应的层间距 为 $0.706 \mathrm{~nm}$ 。当加入 $\mathrm{AN}$ 后, $\mathrm{AGO}$ 的(001)面衍射角 变小 $\left(6.23^{\circ}\right)$, 表明附着 $\mathrm{AN}$ 的 $\mathrm{GO}$ 片层间距明显增 大。经过原位聚合和诱导自组装后所得的 PGO 层间 距同样大于 $\mathrm{GO}$, 其中 $\mathrm{PGO} 0.5$ 与 $\mathrm{PGO} 1$ 的层间距分 别为 0.93 和 $1.18 \mathrm{~nm}$, 说明 $\mathrm{AN}$ 含量越多, 则 $\mathrm{PGO}$ 的层间距越大。经过炭化处理后的样品 GR、 PGR0.5、PGR1 分别在 $2 \theta=26.57^{\circ} 、 25.91^{\circ}$ 和 $25.78^{\circ}$ 出现了新的衍射峰, 对应于有序堆叠石墨烯(002)面 的特征峰, 层间距分别为 $0.335 、 0.343$ 和 $0.345 \mathrm{~nm}$ 。 PGR 的层间距随 AN 含量增加而增大, 但与未添加 聚苯胺的 GR 相比, PGR 的(002)面衍射峰变宽, 表明 存在聚苯胺炭时, 石墨烯片层堆叠的规整度降低。

从图 3 的 SEM 照片可以看出, $\mathrm{GO}$ (图 3(a))与 GR(图 3(e))表面光滑并呈卷曲结构。PANI(图 3(d)) 由直径不一的粒子团聚而成, 炭化后所得的聚苯胺

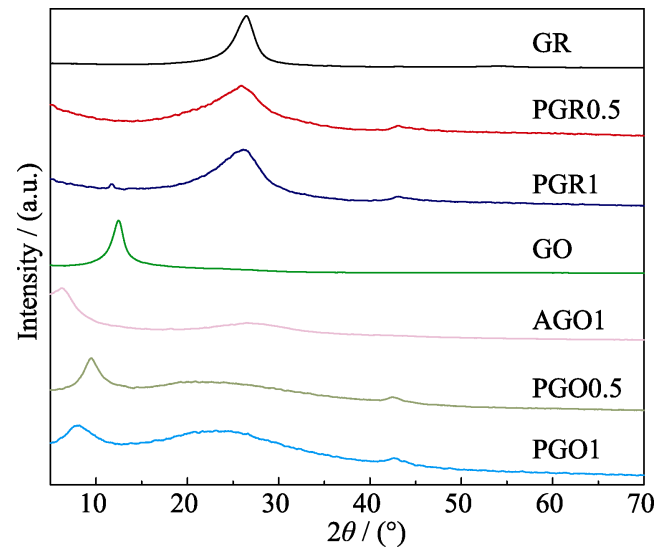

图 $2 \mathrm{GO} 、 \mathrm{AGO} 1 、 \mathrm{PGO} 、 \mathrm{GR}$ 和 PGR 复合材料的 XRD 图谱 Fig. 2 XRD patterns of GO, AGO1, PGOs, GR, and PGRs
炭 CP(图 3(h))依然呈现粒状结构，但由于炭化收缩 作用使得粒径变小。PGO 复合材料(图 3(b)和(c))由 于真空抽滤的诱导自组装作用, 氧化石墨烯呈层状 有序堆叠结构。但与 GO 相比, PGO 复合材料的表 面粗楉, 片层较厚。随着 $\mathrm{AN}$ 含量的增加, $\mathrm{PGO}$ 复合 材料的片层厚度与层间距均增大，与 XRD 结果一 致。PGR 复合材料(图 3(f)和 $(\mathrm{g})$ )的形貌与炭化处理 前的 PGO 复合材料的形貌相似，表面粗龯且堆积更 加疏松，表明聚苯胺炭不仅包覆在石墨烯表面，并 且已通过诱导自组装作用引入石墨烯片层间形成柱 撑结构。这种结构有利于 $\mathrm{Li}^{+}$的嵌入和脱出, 从而提 高电化学性能。

GR、CP、PGR0.5 和 PGR1 的 TEM 照片如图 4 所示, GR(图 4(a))依然表现出光滑的薄片卷曲结构, 图 4(d)为 $\mathrm{CP}$ 的 $\mathrm{TEM}$ 照片, 可见 $\mathrm{CP}$ 是由直径为
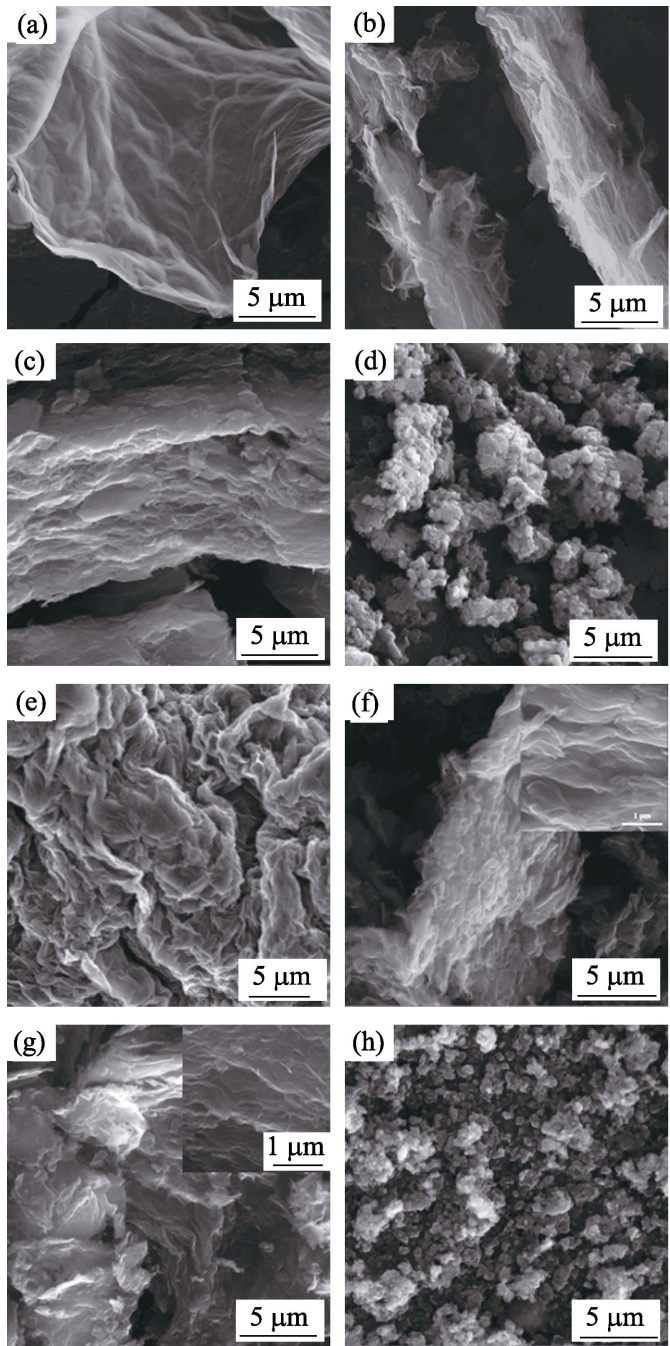

图 3 (a) GO, (b) PGO0.5, (c) PGO1, (d) PANI, (e) GR, (f) PGR0.5, (g) PGR1 和(h) CP 的 SEM 照片

Fig. 3 SEM images of (a) GO, (b) PGO0.5, (c) PGO1, (d) PANI, (e) GR, (f) PGR0.5, (g) PGR1 and (h) CP with insets showing magnified photos 


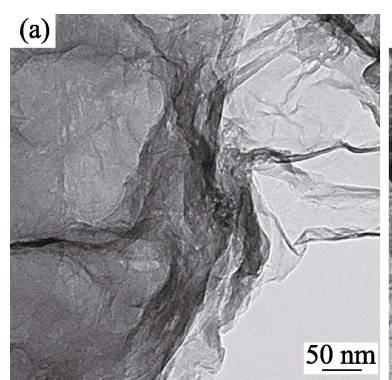

(b)

(c)
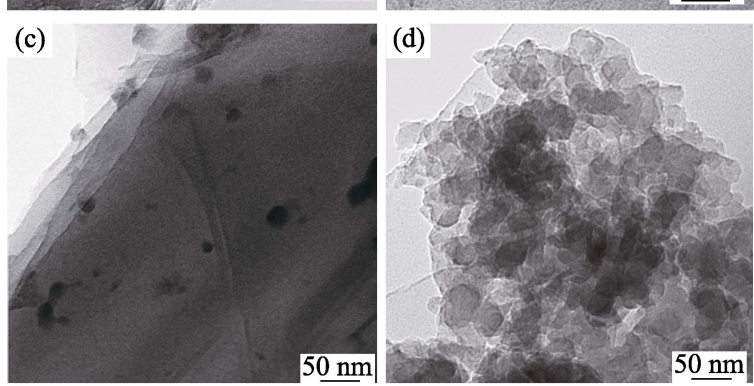

图 4 (a) GR、(b) PGR0.5、(c) PGR1 和(d) CP 的 TEM 照片

Fig. 4 TEM images of (a) GR, (b) PGR0.5, (c) PGR1, and (d) CP

20 30 nm 的粒子团聚形成的无定形颗粒。由图 4(b) (c) 可以看出, PGR 仍表现为薄片层状结构, 且能明显 看到聚苯胺炭均匀地分散于 GR 表面及片层中。当 AN 含量提高时，在 PGR1 片层之间出现了更多的柱 撑聚苯胺炭(图 4(c))。结合 XRD 与 SEM 照片可说 明, PGO 能通过诱导自组装作用将 PANI 引入 GO 片 层之间，炭化处理后形成具有柱撑结构的 PGR 复合 材料。

\section{2 组分分析}

图 5(a)为 GO、PANI、PGO0.5 和 PGO1 的红外 光谱图, 从图中可以看出, $\mathrm{GO}$ 存在较强的特征峰, 1730 和 $1619 \mathrm{~cm}^{-1}$ 分别是 $\mathrm{C}=\mathrm{O}$ 键和 $\mathrm{C}=\mathrm{C}$ 键的伸缩 振动峰。在 PANI 的 FT-IR 谱图上, 1564 和 $1483 \mathrm{~cm}^{-1}$ 的红外特征峰分别为醌环的 $\mathrm{C}-\mathrm{N}$ 振动和苯环的 $\mathrm{C}=\mathrm{C}$ 振动 ${ }^{[25]}$ 。 $\mathrm{PGO}$ 复合材料的 FT-IR 谱中不仅包 含了 PANI 与 GO 的所有特征峰, 而且 AN 含量越
高，与 PANI 的 FT-IR 谱相似度越高。结果表明成 功制备出聚苯胺/氧化石墨烯复合材料。相对于纯 PANI 而言, PGO 复合材料的特征峰分别红移至 1560 和 $1479 \mathrm{~cm}^{-1}$, 且峰强变弱。产生红移和峰强 变弱的主要原因是由于 $\mathrm{GO}$ 平面和 PANI 链之间的 $\pi-\pi$ 作用 ${ }^{[26] \text { 。 }}$

GR、PGR0.5 和 PGR1 的拉曼光谱图如图 5(b) 所示。图中 $G R$ 的 $D$ 峰和 $G$ 峰分别位于 1328 与 $1584 \mathrm{~cm}^{-1}$ 。与 $\mathrm{GR}$ 相比, PGR1 的 D 峰和 $\mathrm{G}$ 峰移至 1318 与 $1576 \mathrm{~cm}^{-1}$, 峰宽变大, 且 $\mathrm{D}$ 峰和 $\mathrm{G}$ 峰强度 比 $\left(I_{\mathrm{D}} / I_{\mathrm{G}}\right)$ 为 1.25 , 高于 $\mathrm{GR}$ 的 1.11 , 表明经过炭化热 处理, PGR1 中的石墨烯已恢复其共轭结构 ${ }^{[27]}$, 但由 于聚苯胺炭柱撑在石墨烯层间导致其无序度增加。

PGR0.5 也有类似的变化。

为了确定样品的元素组成及存在形式, 对 GR、 $\mathrm{CP}$ 和 PGR 进行了 XPS 测试, 结果如图 6(a)所示。 $\mathrm{GR}$ 样品在 284.92 和 $533.16 \mathrm{eV}$ 处分别存在较强的 $\mathrm{C} 1 \mathrm{~s}$ 峰和较弱的 $\mathrm{O} 1 \mathrm{~s}$ 峰, 表明热处理可以很大程度 地还原 GO, 但仍然存在少量的含氧官能团。CP 样 品在 $400.2 \mathrm{eV}$ 处存在较强的 $\mathrm{N} 1 \mathrm{~s}$ 峰, 经计算其 $\mathrm{N}$ 元 素的原子含量为 $7.23 \%$ 。 PGR 样品在 $400.64 \mathrm{eV}$ 处 也存在明显的 N1s 峰, PGR0.5 与 PGR1 的 N 元素的 原子含量分别为 $3.96 \%$ 和 $4.49 \%$, 表明添加 PANI 可 在复合体系中引入较多的 $\mathrm{N}$ 元素。对 PGR1 样品的 N1s 峰进行分峰拟合, 结果如图 6(b) 所示。PGR1 中 的 $\mathrm{N}$ 以四种形式存在, 398.36、400.71、402.42 和 $405.60 \mathrm{eV}$ 处的 N 1s 峰分别对应吡啶 N、吡咯 N、 石墨型 $\mathrm{N}$ 和氧化型 $\mathrm{N}^{[28]}$, 含量分别为 $33.3 \%$ 、44.1\%、 $16.6 \%$ 和 $6 \%$ 。由于吡啶 $\mathrm{N}$ 与吡咯 $\mathrm{N}$ 具有较高的电 化学活性, 有利于改善石墨烯的储锂性能 ${ }^{[29]}$, 而 PGR1 具有较高含量的吡啶 $\mathrm{N}$ 与吡咯 $\mathrm{N}$ (总含量接近 $80 \%$ ), 因此为提高其作为锂电池负极材料的可逆比 容量提供了有利条件。
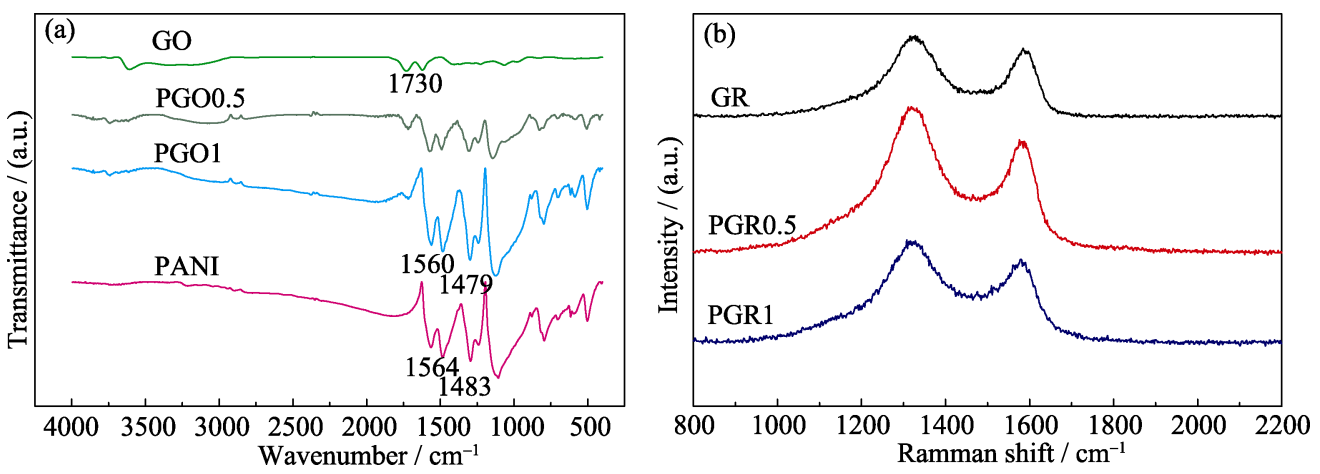

图 5 (a) GO、PGO 和 PANI 的红外光谱图; (b) GR 和 PGR 的拉曼光谱图

Fig. 5 (a) FT-IR spectra of GO, PGOs and PANI samples, and (b) Raman spectra of GR and PGRs samples 

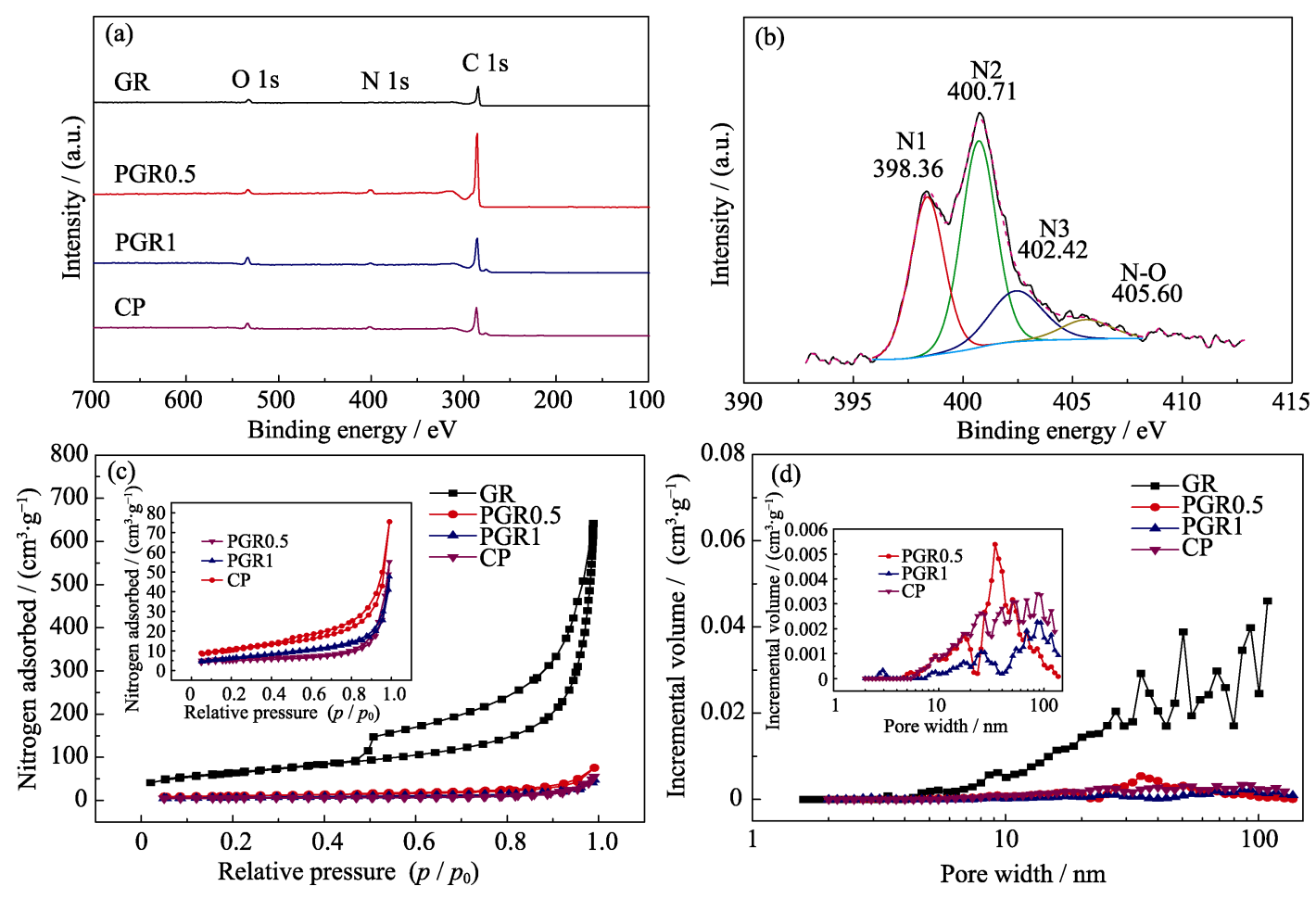

图 6 (a) GR、PGR0.5、PGR1 和 CP 的 XPS 图谱; (b) PGR1 的 N1s 峰的分峰曲线;

(c) GR、PGR0.5、PGR1 和 CP 的氮气吸脱附等温线和(d)孔径分布图

Fig. 6 (a) XPS general spectra of GR, PGRs and CP, (b) curve fitting of N1s spectrum of the sample PGR1, (c) nitrogen sorption isotherms, and (d) DFT pore size distributions of GR, PGRs and CP

为进一步考察孔结构与比表面积的影响, 测试了 样品 GR、CP 和 PGR 的氮气吸脱附等温线, 如图 6(c) 所示。根据 BET 理论计算得知, GR 的比表面积高达 $224 \mathrm{~m}^{2} / \mathrm{g}$, 而 PGR0.5 与 PGR1 的比表面积分别为 36 和 $22.3 \mathrm{~m}^{2} / \mathrm{g}$, 略大于 $\mathrm{CP}$ 的比表面积 $\left(17.5 \mathrm{~m}^{2} / \mathrm{g}\right)$, 但 远小于 GR 的比表面积, 因此有利于增加可逆容量, 提高首次库伦效率 ${ }^{[30]}$ 。采用 DFT 模型得到的孔径分 布如图 6(d)所示，四种样品都存在较多的中孔，其 中 GR 的孔径分布较宽, 既存在少量微孔也存在较 多大孔。而 PGR0.5 与 PGR1 的孔径分布相似，均集 中于 $30 \mathrm{~nm}$ 左右, 微孔和大孔均较少。表明由于聚 苯胺炭的柱撑作用, PGR 复合材料孔径适中, 有利 于 $\mathrm{Li}^{+}$在石墨烯层间快速地嵌入与脱出。

\section{3 电化学性能}

样品 GR、PGR0.5 和 PGR 1 在 $0.1 \mathrm{mV} / \mathrm{s}$ 电压扫 描速率下的首次 $\mathrm{CV}$ 曲线如图 7(a)所示, 三种样品 在 $0.6 \mathrm{~V}$ 左右都出现了还原峰, 这是电解液在电极 表面形成 SEI 膜所造成的。0 左右的还原峰对应于 $\mathrm{Li}^{+}$与炭材料的反应 ${ }^{[31]}$ 。由 PGR1 的前三次 $\mathrm{CV}$ 曲线 (图 7(b)) 可以看出, 仅首次放电过程中在 $0.55 \mathrm{~V}$ 出现 还原峰, 说明 PGR1 复合材料能形成较稳定的 SEI 膜，具有良好的循环稳定性。

图 7(c)对比了样品 GR、PGR0.5 和 PGR1 在 $100 \mathrm{~mA} / \mathrm{g}$ 电流密度下的首次充放电性能。可见, 三
种样品首次嵌锂比容量和脱锂比容量分别为 670 和 $340 \mathrm{mAh} / \mathrm{g} 、 724$ 和 $367 \mathrm{mAh} / \mathrm{g} 、 1083$ 和 $653 \mathrm{mAh} / \mathrm{g}$, PGR1 的可逆比容量最高, 远高于 GR 的可逆比容 量。PGR0.5 与 PGR1 的首次库伦效率分别为 $50.6 \%$ 和 $60.3 \%$ ，也均高于 GR(48.6\%)。样品 GR、PGR0.5 和 PGR1 在不同电流密度下的倍率性能如图 7(d)所 示。当电流密度逐渐增大时, 三种样品的可逆比容 量均有所衰减, 但 $\mathrm{GR}$ 衰减更快。在 $1 \mathrm{~A} / \mathrm{g}$ 的电流密 度下, GR 的可逆比容量仅为 $101 \mathrm{mAh} / \mathrm{g}$, 而 PGR1 的可逆比容量仍高达 $343 \mathrm{mAh} / \mathrm{g}$, 达到 GR 的 3 倍以 上。诱导自组装形成的柱撑结构可以在石墨烯层间 产生大量的拓扑缺陷，提高 $\mathrm{Li}^{+}$的迁移速率。此外， 较高含量的吡啶型 $\mathrm{N}$ 与吡咯型 $\mathrm{N}$ 有利于 $\mathrm{Li}^{+}$的传输, 从而, PGR 复合材料表现出优异的倍率性能 ${ }^{[32]}$ 。

为进一步考察 GR 与 PGR 的电化学特性, 在 3 次 $\mathrm{CV}$ 循环之后对电池进行了交流阻抗测试。如图 8 所示，从图中可以看出 Nyquist 曲线分为三个部分。 其中, 高频区半圆部分主要与电池内部电阻、SEI 膜电阻有关; 中频区半圆主要与电池内部电荷转移 阻抗有关; 而低频区斜线主要与 $\mathrm{Li}^{+}$在电极固相内 的扩散过程有关。通过 ZSimp Win 软件对三条 Nyquist 曲线进行模拟, 得到一个合适的等效电路, 如图 8 插图(实线为拟合数据)。 $R_{\mathrm{s}}$ 代表电池内部电 阻, $R_{\text {sei }}$ 代表界面膜阻抗, $R_{\mathrm{ct}}$ 代表电荷转移阻抗, $Z_{\mathrm{W}}$ 

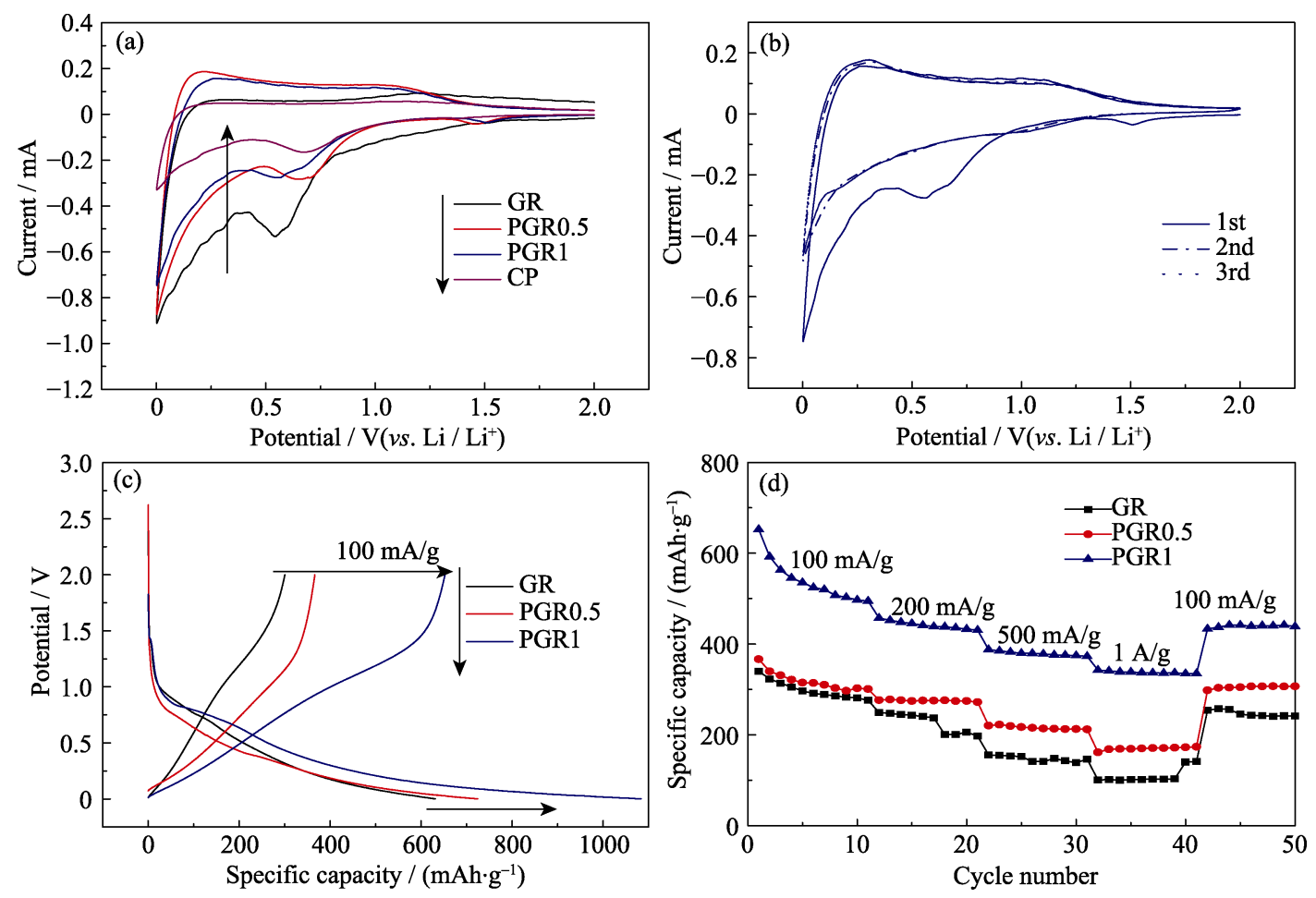

图 7 GR、PGR0.5 和 PGR1 的电化学性能

Fig. 7 Electrochemical performance of GR, PGR0.5, and PGR1

(a) The first CV curve of the samples; (b) The first to the third CV curves of PGR1; (c) The first charge/discharge profile cycle and (d) rate capability

代表 $\mathrm{Li}^{+}$在固相内扩散的 Warburg 阻抗。模拟得到的 各交流阻抗值见表 1 。从表中可以看出, 三种负极材 料的 $R_{\mathrm{S}}$ 差异不大, 但 $R_{\mathrm{sei}}$ 和 $R_{\mathrm{ct}}$ 的差异很明显。说明 PGR 复合材料材料中起柱撑作用的聚苯胺炭, 能够形 成有效的三维导电网络, 提高材料导电性, 并且形 成的空隙能为电解液浸润、 $\mathrm{Li}^{+}$的快速传输提供通道,

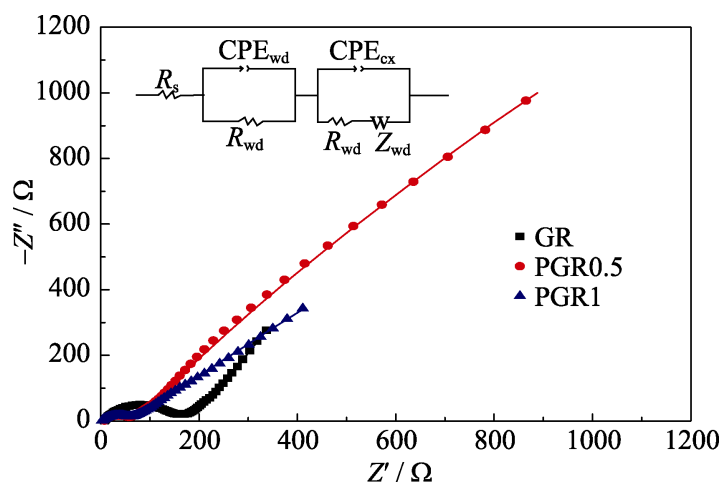

图 8 GR、PGR0.5 和 PGR1 的交流阻抗图谱

Fig. 8 Nyquist plots for the samples of GR, PGR0.5, and PGR1

表 1 GR、PGR0.5 和 PGR1 负极材料的交流阻抗拟合数据 Table 1 EIS fitting results of GR, PGR0.5, and PGR1 electrodes

\begin{tabular}{cccc}
\hline Sample & $R_{\mathrm{s}} / \Omega$ & $R_{\text {sei }} / \Omega$ & $R_{\mathrm{ct}} / \Omega$ \\
\hline GR & 7.23 & 141.3 & 90.67 \\
PGR0.5 & 6.03 & 47.13 & 82.87 \\
PGR1 & 2.02 & 54.50 & 0.10 \\
\hline
\end{tabular}

因此传输阻力明显减小, 表现出优异的倍率性能。

\section{3 结论}

通过静电吸引、 $\pi-\pi$ 共轭以及氢键等作用, 经过 原位聚合及真空抽滤诱导自组装, 在氧化石墨烯中 均匀引入聚苯胺，再通过热解还原，可制备出具有 柱撑结构的聚苯胺炭/石墨烯复合材料。与纯石墨烯 样品相比, $\mathrm{AN}$ 与 $\mathrm{GO}$ 质量比为 $1: 1$ 时制备的 PGR1 复合材料，层间距增大至 $0.345 \mathrm{~nm}$, 氮原子含量达 $4.49 \%$ 。在 $100 \mathrm{~mA} / \mathrm{g}$ 电流密度下的首次脱锂比容量 为 $653 \mathrm{mAh} / \mathrm{g}$, 在 $1 \mathrm{~A} / \mathrm{g}$ 的电流密度下的脱锂比容量 仍高达 $343 \mathrm{mAh} / \mathrm{g}$ ，远超过纯石墨烯样品脱锂比容量 $(101 \mathrm{mAh} / \mathrm{g})$, 表现出优异的倍率性能。聚苯胺炭的 柱撑作用明显提高了有序堆叠石墨烯的电化学性能。

\section{参考文献:}

[1] NOVOSELOV K S, GEIM A K, MOROZOV S V, et al. Two-dimensional gas of massless Dirac fermions in graphene. $\mathrm{Na}$ ture, 2005, 438(7065): 197-200.

[2] HAN P X, YUE Y H, ZHANG L X, et al. Nitrogen-doping of chemically reduced mesocarbon microbead oxide for the improved performance of lithium ion batteries. Carbon, 2012, 50(3): $1355-1362$.

[3] LI D, MULLER M B, GILJE S, et al. Processable aqueous dispersions of graphene nanosheets. Nat. Nanotechnol., 2008, 3(2): 101-105. 
[4] WANG G X, SHEN X P, YAO J, et al. Graphene nanosheets for enhanced lithium storage in lithium ion batteries. Carbon, 2009, 47(8): 2049-2053.

[5] BONACCORSO F, SUN Z, HASAN T, et al. Graphene photonics and optoelectronics. Nat. Photonics, 2010, 4(9): 611-622.

[6] WAN L J, REN Z Y, WANG H, et al. Graphene nanosheets based on controlled exfoliation process for enhanced lithium storage in lithium-ion battery. Diam. Relat. Mater., 2011, 20(5/6): 756-761.

[7] LEE S H, SEO S D, PARK K S, et al. Synthesis of graphene nanosheets by the electrolytic exfoliation of graphite and their direct assembly for lithium ion battery anodes. Mater. Chem. Phys., 2012, 135(2/3): 309-316.

[8] SANG Y, ZHOU Y, XIE H, et al. Assembling and nanocutting graphene/CNT sponge for improved lithium-ion batteries. Ionics, 2017, 23(5): 1329-1336.

[9] KIM C, KIM J W, KIM H, et al. Graphene oxide assisted synthesis of self-assembled zinc oxide for lithium-ion battery anode. Chemistry of Materials, 2016, 28(23): 8498-8503.

[10] ZHANG Q Q, LI R, ZHANG M M, et al. $\mathrm{TiO}_{2}$ nanocrystals/ graphene hybrids with enhanced Li-ion storage performance. $J$. Energy Chem., 2014, 23(3): 403-410.

[11] LI L, RAJI A R, TOUR J M. Graphene-wrapped $\mathrm{MnO}_{2}$-graphene nanoribbons as anode materials for high-performance lithium ion batteries. Advanced Materials, 2013, 25(43): 6298-6302.

[12] PING, G Z, ZHANG J Y, CHENG J, et al. Graphene nanosheets prepared by low-temperature exfoliation and reduction technique toward fabrication of high-performance poly(1-butene)/graphene films. Iran. Polym. J., 2017, 26(1): 55-69.

[13] SHEN B, LU D D, ZHAI W T, et al. Synthesis of graphene by low-temperature exfoliation and reduction of graphite oxide under ambient atmosphere. J. Mater. Chem. C, 2013, 1(1): 50-53.

[14] MENG L Y, PARK S J. Synthesis of graphene nanosheets via thermal exfoliation of pretreated graphite at low temperature. $A d v$. Mater. Res-Switz, 2010, 123-125: 787-790.

[15] NIU S Z, LV W, ZHANG C, et al. One-pot self-assembly of graphene/ carbon nanotube/sulfur hybrid with three dimensionally interconnected structure for lithium-sulfur batteries. Journal of Power Sources, 2015, 295: 182-189.

[16] WU H W, HUANG Y, ZONG M, et al. Electrostatic self-assembly of graphene oxide wrapped sulfur particles for lithium-sulfur batteries. Mater. Res. Bull., 2015, 64: 12-16.

[17] TANG J J, YANG J, ZHOU L M, et al. Layer-by-layer selfassembly of a sandwich-like graphene wrapped $\mathrm{SnO}_{x} @$ graphene composite as an anode material for lithium ion batteries. J. Mater. Chem. A, 2014, 2(18): 6292-6295.

[18] BAI X J, LIU C, HOU M, et al. Silicon/CNTs/graphene free-standing anode material for lithium-ion battery. Journal of Inorganic Materials, 2017, 32(7): 705-712.

[19] HU A P, CHEN X H, TANG Y H, et al. Self-assembly of $\mathrm{Fe}_{3} \mathrm{O}_{4}$ nanorods on graphene for lithium ion batteries with high rate capacity and cycle stability. Electrochemistry Communications, 2013, 28: $139-142$.

[20] ZHAO M Q, LIU X F, ZHANG Q, et al. Graphene/single-walled carbon nanotube hybrids: one-step catalytic growth and applications for high-rate Li-S batteries. ACS Nano, 2012, 6(12): 10759-10769.

[21] KUMAR N A, CHOI H J, SHIN Y R. Polyaniline-grafted reduced graphene oxide for efficient electrochemical supercapacitors. ACS Nano, 2012, 6(2): 1715-1723.

[22] ZHANG K, ZHANG L L, ZHAO X S, et al. Graphene/polyaniline nanofiber composites as supercapacitor electrodes. Chemistry of Materials, 2010, 22(4): 1392-1401.

[23] ZHU B Y, DENG Z, YANG W L, et al. Pyrolyzed polyaniline and graphene nano sheet composite with improved rate and cycle performance for lithium storage. Carbon, 2015, 92: 354-361.

[24] HUMMERS W S, OFFEMAN R E. Preparation of graphitic oxide, J. Am. Chem. Soc., 1958, 80: 1339-1339.

[25] YANG F, XU M, BAO S J, et al. Self-assembled hierarchical graphene/ polyaniline hybrid aerogels for electrochemical capacitive energy storage. Electrochimica Acta, 2014, 137: 381-387.

[26] SUN Y B, SHAO D, CHEN C, et al. Highly efficient enrichment of radionuclides on graphene oxide-supported polyaniline. Environmental Science \& Technology, 2013, 47(17): 9904-9910.

[27] FAN W, XIA Y Y, TJIU W W, et al. Nitrogen-doped graphene hollow nanospheres as novel electrode materials for supercapacitor applications. Journal of Power Sources, 2013, 243: 973-981.

[28] WANG C, WANG Y L, ZHAN L, et al. Synthesis of nitrogen doped graphene through microwave irradiation. Journal of Inorganic Materials, 2012, 27(2): 146-150.

[29] LI X, GENG D, ZHANG Y, et al. Superior cycle stability of nitrogendoped graphene nanosheets as anodes for lithium ion batteries. Electrochemistry Communications, 2011, 13(8): 822-825.

[30] WANG D W, LI F, LIU M, et al. 3D aperiodic hierarchical porous graphitic carbon material for high-rate electrochemical capacitive energy storage. Angew. Chem. Int. Ed., 2008, 47(2): 373-376.

[31] LI X F, GENG D S, ZHANG Y, et al. Superior cycle stability of nitrogen-doped graphene nanosheets as anodes for lithium ion batteries. Electrochemistry Communications, 2011, 13: 822-825.

[32] WANG Z, CHEN T, CHEN W X, et al. CTAB-assisted synthesis of single-layer $\mathrm{MoS}_{2}$-graphene composites as anode materials of Li-ion batteries. J. Mater. Chem. A, 2013, 1(6): 2202-2210. 